\title{
Increased Stress Tolerance of Transgenic Tobacco Plants that Overexpress Ascorbate Peroxidase in Chloroplasts
}

\author{
Hironori Kaminaka ${ }^{1}$, Shigeto Morita ${ }^{1,2}$, Toru Suzuki ${ }^{1}$, Hidefumi \\ Yokoyama ${ }^{1}$, Akihiro Kubo ${ }^{3}$, Takehiro Masumura ${ }^{1,2}$, Hikaru Saji ${ }^{3}$, \\ Kiyoshi Tanaka ${ }^{4}$ \& Kunisuke Tanaka ${ }^{1,2}$ \\ 1. Laboratory of Genetic Engineering, Faculty of Agriculture, Kyoto \\ Prefectural University, Kyoto 606-8522, Japan \\ 2. Kyoto Prefectural Institute of Agricultural Biotechnology, Seika, Kyoto \\ 619-0244, Japan \\ 3. Environmental Biology Division, National Institute for Environmental \\ Studies, \\ 4. Faculty of Agriculture, Tottori University
}

Because of their inability to move, plants are invariably exposed to various environmental stresses such as extreme temperatures, high light and salinity, which affect growth and productivity of plants. One of the major causes of damages by those stresses is photooxidative injuries by active oxygen species. In higher plants, superoxide dismutase (SOD) and ascorbate peroxidase (APX) play central roles in active oxygen scavenging system. In order to clarify the correlation between these two enzymes and stress tolerance, we produced transgenic tobacco plants that overexpress SOD or APX in chloroplasts.

Total SOD activity of the SOD-transgenic plant (PSOD) was increased to 2.3-fold compared with wild type, with no significant changes in total APX activity. While in the APX transformant (PAPX), total APX activity was elevated to 8.5-fold and total SOD activity was also increased to 1.9 -fold. Therefore, APX overexpression caused the elevation of endogenous SOD activity in PAPX plants, which is probably due to the decrease in cellular hydrogen peroxide level caused by APX overexpression.

We then examined the stress tolerance of these transformants. When subjected to chilling stress under moderate light $\left(220 \mathrm{mmol} / \mathrm{m}^{2} / \mathrm{s}\right)$, foliar damages were developed in wild type plants, but no visible damages were found in both PSOD and PAPX plants in this condition. However, drought stress under high light $\left(1000 \mathrm{mmol} / \mathrm{m}^{2} / \mathrm{s}\right)$ caused severe damage not only in wild type but also in PSOD. Only PAPX plants showed the increased tolerance to drought stress. Measurement of chlorophyll fluorescence of PSII also revealed that PAPX plants were more tolerant to photooxidative damages by methyl viologen treatment than wild type and PSOD plants. These results suggest that the elevation of scavenging capacities of hydrogen peroxide is effective and crucial for stress tolerance. 